\title{
RUPTURED INTRACRANIAL DERMOID CYST
}

\author{
Taimoor Ali Tariq, Pir Abdul Ahad Aziz Qureshi, Muhammad Talha Yaseen Kaimkhani \\ Department of Radiology, Shaukat Khanum Memorial Cancer Hospital and Research Centre, Lahore, Pakistan \\ Received: 30 January 2019/ Accepted: 12 June 2019
}

We present here a case of a 27 -year-old male patient who presented with a history of intermittent seizures for 1 year and was treated for epilepsy. These seizures were not controlled with regular medications. Magnetic resonance imaging (MRI) scan of the brain showed ruptured dermoid cyst with spilling of cyst contents into the temporal horn of the left lateral ventricle and subarachnoid space. No obstructive hydrocephalus was noted [Figure 1].

Dermoid cysts are an uncommon entity of ectodermal origin with reported incidence of $0.4-0.6 \%$ of all intracranial tumours. It contains sweat glands, sebaceous glands and hair follicles. ${ }^{[1,2]}$ These lesions may present with local mass effect, headache or features of meningitis. ${ }^{[3]}$ Dermoid cysts have tendency to cause significant morbidity by compression of the adjacent neurovascular structures, and rarely, these can also rupture with intracystic content disseminating into the subarachnoid

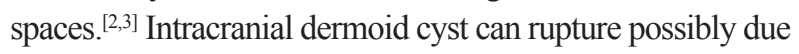
to sudden shift in the cyst's sac secondary to trauma or it can be spontaneous resulting in chemical meningitis. ${ }^{[3]}$ Computed tomography scan is usually adequate for making the diagnosis of ruptured dermoid cyst; however, MRI provides better characterisation of the intracystic contents dissemination which is very important in surgical planning.

At presentation, intravenous steroids are helpful for symptomatic relief in the aseptic meningitis associated with rupture. Complete surgical resection is the aim, but injury to surrounding vital structures can result in fatal outcome. If there is associated hydrocephalus, it should be managed promptly and the patient should be monitored closely in the perioperative period. The recurrence rate with subtotal resection is extremely rare; however, routine follow is recommended. ${ }^{[2]}$

Ruptured dermoid cyst is a rarely reported incident. A presentation with seizures should generally be evaluated radiologically for organic causes. A ruptured dermoid cyst can be completely characterised with routine MRI sequences.

\footnotetext{
Correspondence: Pir Abdul Ahad Aziz Qureshi, Department of Radiology, Shaukat Khanum Memorial Cancer Hospital and Research Centre, Lahore, Pakistan.

Email: abdulahad.q@gmail.com
}
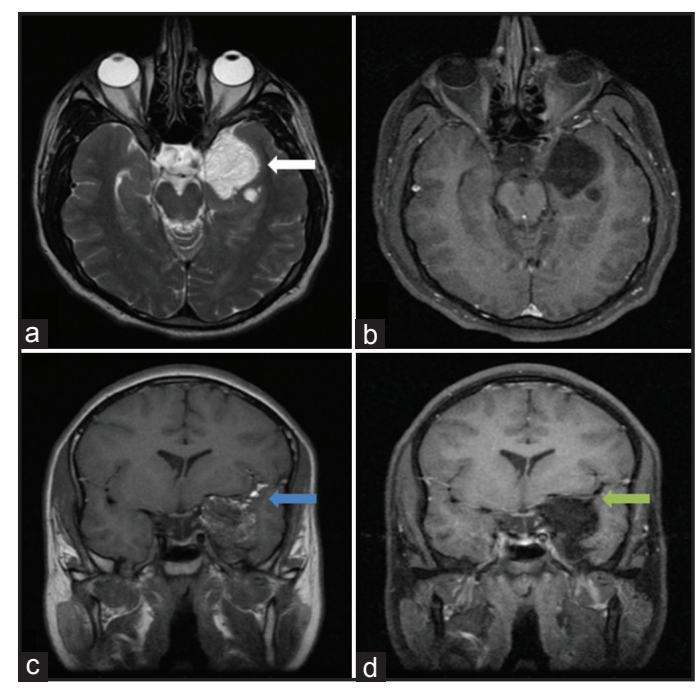

Figure 1: Extra-axial mass in relation to the anteromedial aspect of the left temporal lobe (white arrow). T2W high and T1W intermediate to high signal intensity mass (a and c). There are specks of high signal on T1W imaging (c) (blue arrow). There is complete suppression of all these high signals on post-contrast fat-suppressed images (b and d) (green arrow), indicating fat contents. Findings are compatible with an extra-axial dermoid cyst which has ruptured into the subarachnoid space

\section{Conflict of Interest}

The authors declare that they have no conflict of interest.

\section{References}

1. Murrone D, Romanelli B, Ierardi A. Ruptured intracranial dermoid cyst: A case report. Integr Mol Med 2016;3:793-5.

2. Esquenazi Y, Kerr K, Bhattacharjee MB, et al. Traumatic rupture of an intracranial dermoid cyst: Case report and literature review. Surg Neurol Int 2013;4:80.

3. Roy P, Kucera J, Murtagh R. Ruptured intracranial dermoid cyst manifesting as new onset seizure: A case report. J Radiol Case Rep 2011;5:10-8.

\section{Authorship Contributions}

Concept and Design: TAT, PAAAQ, MTYK; Data Collection and interpretation: TAT, PAAAQ, MTYK; Literature review and drafting: TAT, PAAAQ; Manuscript approval: TAT, PAAAQ, MTYK 